\title{
ON SEMIGROUP PRESENTATIONS
}

\author{
by EDMUND F. ROBERTSON AND YUSUF ÜNLÜ*
}

(Received 4th December 1990)

\begin{abstract}
Semigroup presentations have been studied over a long period, usually as a means of providing examples of semigroups. In 1967 B. H. Neumann introduced an enumeration method for finitely presented semigroups analogous to the Todd-Coxeter coset enumeration process for groups. A proof of Neumann's enumeration method was given by Jura in 1978 .

In Section 3 of this paper we describe a machine implementation of a semigroup enumeration algorithm based on that of Neumann. In Section 2 we examine certain semigroup presentations, motivated by the fact that the corresponding group presentation has yielded interesting groups. The theorems, although proved algebraically, were suggested by the semigroup enumeration program.
\end{abstract}

1980 Mathematics subject classification (1985 Revision): $20 \mathrm{M} 05$.

\section{Definitions and preliminary theorems}

Let $X$ be a finite set and let $F_{S}(X)$ be the free semigroup (excluding the empty word) on $X$. Throughout this paper, by a presentation $P$ on $X$ we mean a generating set $X$ together with a finite set of relations $L_{i}=R_{i}, i \in I$ where $L_{i}, R_{i} \in F_{S}(X)$. We write

$$
P=\left\langle X: L_{i}=R_{i}, i \in I\right\rangle
$$

and use the notation Smgp $P$ and $G p P$ for the free-est semigroup on $X$ satisfying the relations $L_{i}=R_{i}, i \in I$ and the free-est group on $X$ satisfying the relations $L_{i}=R_{i}, i \in I$, respectively. We are thus only interested in finitely presented semigroups and finitely presented groups. We use the symbols $\mathbb{N}$ and $\mathbb{Z}$ for the set of positive integers and the set of integers, respectively.

Definition 1.1. Let $P$ be a presentation on $X$. We say that $S m g p P$ has a group kernel if it has an ideal which is isomorphic to $G p P$.

Theorem 1.2. Let $P$ be a presentation, $S=\operatorname{Smgp} P$ and $G=G p P$.

(a) $S$ has a group kernel if and only if $S$ has an idempotent $e$ such that $S e$ is a group which is an ideal in $S$.

*Supported by the Turkish Scientific and Technical Research Council Mathematics Research Group (TBAG-C2). 
(b) If Se and $S f$ are group kernels in $S$ for idempotents $e$ and $f$, then $e=f$. In particular $S$ can only have one group kernel.

Proof. (a) Let $I$ be a group kernel in $S$. If $e$ is the identity of $I$, then $e$ is an idempotent in $S$. We have

$$
S e \subset I=I e \subset S e
$$

which implies that $I=S e$.

Conversely, suppose that $e \in S$ is an idempotent such that $S e$ is a group which is an ideal in $S$. Then $e$ is the identity of $S e$. For $w \in S$ let us define $\tilde{w}=w e$. If $w, u \in S$ then

$$
\tilde{w} \tilde{u}=(w e)(u e)=w(e(u e))=w(u e)=(w u) e=(\tilde{w u}) .
$$

Hence if $x_{1}, \ldots, x_{n} \in S$ and $w=x_{1} \ldots x_{n}$ then $\tilde{w}=\tilde{x}_{1} \ldots \tilde{x}_{n}$. This shows that $S e$ is generated by $\tilde{X}=\{\tilde{x}: x \in X\}$. Let

$$
P=\left\langle X: L_{1}=R_{1}, \ldots, L_{k}=R_{k}\right\rangle .
$$

As observed above, for $1 \leqq i \leqq k$ we have $\tilde{L}_{i}=\tilde{R}_{i}$. Since $G$ is the group presented by $P$, there is a homomorphism $f$ from $G$ onto $S e$ such that $f(x)=\tilde{x}$. Considering $G$ as a semigroup, we see that there is a homomorphism $g$ from $S$ into $G$ such that $g(x)=x$. Since $e$ is an idempotent, then so is $g(e)$. Thus $g(e)$ is the identity of $G$ and for $x \in X$,

$$
g(\tilde{x})=g(x e)=g(x) g(e)=x
$$

This shows that $g f \mid X=\mathbb{1}_{X}$ and $f g \mid \tilde{X}=\mathbb{1}_{\tilde{X}}$. Consequently $f$ is an isomorphism and $S e$ is a group kernel in $S$.

(b) Let $E=S e$ and $F=S f$. Then $I=E F$ is an ideal in $S$ contained both in $E$ and $F$. Since $e$ and $f$ are the identities of $E$ and $F$, respectively, we have

$$
(e f)(e f)=((e f) e) f)=(e f) f=e f .
$$

This shows that $e f$ is an idempotent in $E \cap F$. So $e=f=e f$.

We can give conditions on a presentation $P=\langle X: R\rangle$, where $X=\left\{x_{1}, x_{2}, \ldots, x_{n}\right\}$, under which $\operatorname{Smgp} P$ will have a group kernel. First we introduce some notation.

Definition 1.3. For $w \in F_{S}(X)$ define $g(w)=\left\{x_{i} \in X: x_{i}\right.$ occurs in $\left.w\right\}$.

Suppose $S=\operatorname{Smg} p P$ is finite and $x_{i}$ satisfies $x_{i}^{m_{i}+1}=x_{i}$ for $1 \leqq i \leqq n$. Then $x_{i}^{m_{i}}$ is idempotent for $1 \leqq i \leqq n$. Consider the conditions:

C1. $x_{i}^{m_{i}+1}=x_{i}$ and $x_{i}^{m_{i}}$ is central in $S$ for $1 \leqq i \leqq n$.

C2. For each relation $L_{i}=R_{i}$ in $R, g\left(L_{i}\right)=g\left(R_{i}\right)$.

Theorem 1.4. If $P=\langle X: R\rangle$ satisfies conditions $\mathrm{C} 1$ and $\mathrm{C} 2$ and $S=\operatorname{Smgp} P$, then $S$ has a group kernel $K_{X}=\{w \in S: g(w)=X\}$. Moreover 


$$
S=\bigcup_{Y} K_{Y}
$$

where the union is taken over all $Y \subseteq X$.

Proof. Clearly $K_{X}$ is an ideal of $S$. Using $\mathrm{C} 1$ we see that the idempotent element

$$
e=x_{1}^{m_{1}} x_{2}^{m_{2}} \ldots x_{n}^{m_{n}}
$$

is the identity of $K_{X}$ and that the inverse of $w=x_{i_{1}} x_{i_{2}} \ldots x_{i_{k}}$, where $g(w)=X$, is

$$
x_{i_{k}}^{m_{i_{k}-1}} x_{i_{k}=1}^{m_{k_{1}-1}{ }^{-1}} \ldots x_{i_{1}}^{m_{i_{1}-1}} \text {. }
$$

Taking any subset $Y$ of $X$ we see that $S_{Y}=\{w \in S: g(w) \subseteq Y\}$ is a subsemigroup of $S$ with group kernel $K_{Y}$ by the above argument. The final conclusion now follows.

The condition $\mathrm{C} 2$ above ensures that $K_{Y} \cap K_{Z}=\varnothing$ for subsets $Y \neq Z$ of $X$. The next result gives similar results under slightly different conditions.

Theorem 1.5. Let $X=\{x, y\}$ where $x \neq y$. Let $A=F_{S}(X) \backslash\left\{x^{i}: i \in \mathbb{N}\right\}$ and let $L_{i}, R_{i} \in A$ for $1 \leqq i \leqq s$. Consider the presentation

$$
P=\left\langle X: x^{n}=w, L_{1}=R_{1}, \ldots, L_{s}=R_{s}\right\rangle
$$

where $w \in F_{S}(X)$ and $n \geqq 2$ is a positive integer. If either $w \in A$ or $w=x$ then in $S=S m g p P$, the elements $x, x^{2}, \ldots, x^{n-1}$ are pairwise distinct and

$$
A \cap\left\{x, x^{2}, \ldots, x^{n-1}\right\}=\varnothing
$$

Proof. Case 1. $\boldsymbol{w} \in \boldsymbol{A}$. Let $B$ be the set of integers $k$ such that $0 \leqq k \leqq 2^{n}-1$. Let us define the functions $f: B \rightarrow B$ and $g: B \rightarrow B$ as $k f=[k / 2]$ and $k g=0$ for $k \in B$, where [k/2] is the integer part of $k / 2$. It is fairly easy to see that

$$
\left(2^{n}-1\right) f^{i}=2^{n-i}-1 \quad \text { for } 1 \leqq i \leqq n .
$$

In particular $f^{n}=g$ and $0 f=0$. Let us observe that if $h: B \rightarrow B$ is any function with $0 h=0$, then $g h=h g=g$. Let $U$ be the subsemigroup of all self-maps of $B$ generated by $f, g$. By the remark we have just made

$$
U=\left\{f, f^{2}, \ldots, f^{n-1}\right\} \cup\{g\} .
$$

There is a homomorphism $\psi$ from $F_{s}(X)$ onto $U$ satisfying $\psi(x)=f, \psi(y)=g$. We note that for all $u \in A, \psi(u)=g$. It follows that

$$
\psi\left(L_{i}\right)=g=\psi\left(R_{i}\right) \quad \text { for } 1 \leqq i \leqq s,
$$


and $\psi(w)=g=f^{n}=\psi\left(x^{n}\right)$. Hence $U$ is a semigroup satisfying the given relations. Consequently there is a homomorphism $\alpha: S \rightarrow U$ such that $\alpha(x)=f$ and $\alpha(y)=g$. By 1.5.1

$$
\alpha(A) \cap \alpha\left(\left\{x, x^{2}, \ldots, x^{n-1}\right\}\right)=\{0\} \cap\left\{f, f^{2}, \ldots, f^{n-1}\right\}=\varnothing
$$

and $f, f^{2}, \ldots, f^{n}$ are pairwise distinct. This shows that

$$
A \cap\left\{x, x^{2}, \ldots, x^{n-1}\right\}=\varnothing
$$

and $x, x^{2}, \ldots, x^{n-1}$ are pairwise distinct.

Case 2. $w=x$. Let $B$ be the set of integers $k$ such that $0 \leqq k \leqq n$. Let us define the functions $f: B \rightarrow B$ and $g: B \rightarrow B$ as

$$
k f= \begin{cases}0 & k=0 \\ k+1 & 0<k<n \\ 2 & k=n\end{cases}
$$

and

$$
k g=0
$$

for $k \in B$. It is easy to see that $f^{n}=f$ and $f, f^{2}, \ldots, f^{n-1}$ are pairwise distinct. Now reasoning as in Case 1 we can prove the assertion.

The following theorem is easy to prove.

Theorem 1.6. Let $P$ be a presentation on $X$ and $S=S m g p P$. Let $H$ be a subset of $S$ such that $X \subseteq H$ and $H x \subseteq H$ for all $x$ in $X$. Then $H=S$.

\section{Some semigroup presentations}

In this section we investigate some semigroup presentations and the relationship between the semigroup and the group presented by the same presentation. First we consider a presentation $P$ where $G p P$ is the dihedral group.

Theorem 2.1. Let $P=<a, b: a^{3}=a, b^{n+1}=b, b a b=a>$ where $n \geqq 2, D S_{n}=\operatorname{Smgp} P$ and $D_{n}=G p P$. Then
(a) $a b^{n}=b^{n} a=a$.
(b) $a^{2} b=b a^{2}=a b^{n-1} a$.
(c) $D S_{n} a^{2}$ is the group kernel of $D S_{n}$ and $\left|D S_{n}\right|=3 n=\left|D_{n}\right|+n$. 
Proof. (a) $b^{n} a=b^{n} b a b=b^{n+1} a b=b a b=a=b a b=b a b^{n+1}=b a b b^{n}=a b^{n}$.

(b) $a^{2} b=a^{2} b^{n} b=a b^{n-1} b a b=a b^{n-1} a=b a b b^{n-1} a=b a b^{n} a=b a^{2}$.

(c) Let $S=D S_{n}$ and $I=S a^{2}$. Now $I$ is an ideal in $S$ by (b) and contains the idempotent $e=a^{2}$. Also, again using (b), we see that $e$ is the identity element of $I$ and

$$
(a e)^{2}=e \quad \text { and } \quad(b e)^{n}=\left(b a^{2}\right)^{n}=b^{n} a^{2}=a^{2}=e .
$$

Thus $a e$ and be have inverses in $S$, which shows that $I$ is a group. So $I$ is the group kernel which is isomorphic to $D_{n}$. If $B=\left\{b, b^{2}, \ldots, b^{n}\right\}$, then $B \cap I=B \cap S a^{2}=\varnothing$ and $b, b^{2}, \ldots, b^{n}$ are pairwise distinct, by Theorem 1.5. Since $a e=a^{3}=a$, then $a \in I$. If $w$ is a word in $S$, then either $w \in B$ or $w$ contains the letter $a$. Consequently $S=B \cup I$. This shows that

$$
|S|=n+\left|D_{n}\right|=3 n
$$

since $D_{n}$ is the dihedral group and $\left|D_{n}\right|=2 n$.

Taking $n=2$ in Theorem 2.1 gives $\left|D S_{2}\right|=6$. However we will show that if we change the second relation from $b^{3}=b$ into $a b^{3}=a b$, the semigroup with this new presentation is infinite. This shows that $G p P$ is not enough to determine the structure of Smgp $P$.

Theorem 2.2. Let $P=\left\langle a, b: a^{3}=a, a b^{3}=a b, b a b=a\right\rangle$. Then $G p P$ is $D_{2}$, the Klein four-group, while Smgp $P$ is infinite.

Proof. Let $a$ and $b$ be the functions from $\mathbb{N}$ to $\mathbb{N}$ defined by

$$
k a=1 \text { and } k b=k^{2} \text { for } k \in \mathbb{N} \text {. }
$$

Then $a^{3}=\mathrm{a}, a b^{3}=a b$ and $b a b=a$. Thus $a$ and $b$ satisfy the given relations. Clearly the powers of $b$ are pairwise distinct, hence $\operatorname{Smg} P P$ is infinite.

The next presentation comes from the presentation for a general metacyclic group.

Theorem 2.3. Let $P=\left\langle x, y: x^{m+1}=x, x y=y x^{r}, y^{n}=x^{s}\right\rangle$ where $m, n, r, s$ are positive integers satisfying

$$
r^{n} \equiv 1(\bmod m), r s \equiv s(\bmod m), \quad s<m, 1<r \leqq m, 2 \leqq n
$$

Let $S=S m g p P$ and $G=G p P$.

(a) $x^{j} y=y x^{j r}$ for all $j \in \mathbb{N}$.

(b) $x^{m} y=y x^{m}$ and $x^{m}$ is an idempotent in $S$.

(c) $S x^{m}$ is the group kernel of $S$ and $|S|=n-1+|G|=n-1+m n$. 
Proof. (a) This easily follows from the relation $x y=y x^{r}$ by induction.

(b) Clearly $x^{m}$ is an idempotent, since $x^{m+1}=x$. We have $x^{m} y=y x^{r m}$ by (a). Since $x^{m}$ is an idempotent, then $x^{k m}=x^{m}$ for all $k$ in $\mathbb{N}$. So $x^{m} y=y x^{m}$.

(c) Since $e=x^{m}$ is central in $S, I=S x^{m}$ is an ideal in $S$ with $e$ as an identity. Since $r s \equiv s(\bmod m)$, then $m \mid(r-1) s$. Hence there is a positive integer $p$ such that $r s=s+m p$. Now

$$
\begin{gathered}
(x e)^{m}=x^{2 m}=x^{m}=e, \\
(y e)^{n(r-1)}=y^{n(r-1)} x^{m}=y^{n(r-1)} x^{s} x^{m-s}=y^{n(r-1)} y^{n} x^{m-s} \\
=y^{n r} x^{m-s}=x^{s r} x^{m-s}=x^{s+m p} x^{m-s}=x^{m(p+1)}=x^{m}=e .
\end{gathered}
$$

It follows that $y e$ and $x e$ each has an inverse in $I$, and so $I$ is a group. Since $x=e x$, then $x \in I$. Reasoning as in Theorem 2.1 , we see that if $Y=\left\{y, y^{2}, \ldots, y^{n-1}\right\}$ then $Y \cap I=$ $Y \cap S x^{m}=\varnothing, S=Y \cup I$ and $y, y^{2}, \ldots, y^{n-1}$ are pairwise distinct. Hence

$$
|S|=n-1+|I|=n-1+m n
$$

since $G$ is a metacyclic group of order $m n$.

Before giving the next theorem, we define the Lucas sequence of numbers $\left\{g_{i}\right\}$ by

$$
g_{0}=2, g_{1}=1, g_{i+2}=g_{i+1}+g_{i} \quad \text { for } i \geqq 0 \text {. }
$$

Theorem 2.4. Let $P=\left\langle a, b: a b^{2}=b a^{2}, a^{n+1}=a, b^{n+1}=b\right\rangle, S=\operatorname{Smgp} P$ and $G=G p P$. Then

(i) if $n$ is odd $|S|=2 n+|G|=2 n+n g_{n}$,

(ii) if $n$ is even then

$$
|S|= \begin{cases}10 & \text { if } n=2 \\ 68 & \text { if } n=4 \\ \infty & \text { if } n \geqq 6 .\end{cases}
$$

That $|S|$ is infinite for $n \geqq 6$ follows since $|G|$ is infinite in this case, see [1]. We shall break the proof of the finite parts of the theorem into a lemma and two examples. We consider first the case where $n$ is odd.

Lemma 2.5. If $S$ and $G$ are as in Theorem 2.4 then, with $n=2 k+1$, we have:

(a) $a b=b a^{2} b^{2 k}$ and $b a=a b^{2} a^{2 k}$;

(b) $a b=(b a)^{k+1} a$ and $b a=(a b)^{k+1} b$; 
(c) $(b a)^{k+1}=a b a^{2 k}$ and $(a b)^{k+1}=b a b^{2 k}$;

(d) $a b=a b a^{2 k+1}$ and $b a=b a b^{2 k+1}$;

(e) $a b=b^{2 k+1} a b$ and $b a=a^{2 k+1} b a$;

(f) $a b^{n}=b^{n} a$ and $b a^{n}=a^{n} b$;

(g) $|S|=2 n+|G|=2 n+n g_{n}$.

Proof. Before we start the proof let us observe that if we interchange $a$ with $b$ in any identity in $S$, then we obtain another identity, since the relations in $P$ are symmetric. So we will prove only one of the identities in each case.

(a) $b a^{2} b^{2 k}=a b^{2} b^{2 k}=a b^{n+1}=a b$.

(b) We prove by induction on $r$ that

$$
a b=(b a)^{r} a b^{2 k-2 r+2} \quad \text { for } 1 \leqq r \leqq k .
$$

$r=1: a b=b a^{2} b^{2 k}=(b a) a b^{2 k-2+2}$.

$r \geqq 1$ : Suppose that $1 \leqq r \leqq k-1$ and the assertion holds for $r$. We have $2 k-2 r>0$.

$$
a b=(b a)^{r} a b^{2} b^{2 k-2 r}=(b a)^{r} b a^{2} b^{2 k-2 r}=(b a)^{r+1} a b^{2 k-2(r+1)+2} .
$$

This completes the induction. If we then take $r=k$ we get

$$
a b=(b a)^{k} a b^{2}=(b a)^{k} b a^{2}=(b a)^{k+1} a .
$$

(c) We prove by induction on $r$ that

$$
(b a)^{k+1}=(b a)^{k-r+1} a b^{2 r} a^{2 k} \text { for } 1 \leqq r \leqq k .
$$

$r=1:(b a)^{k+1}=(b a)^{k} b a=(b a)^{k} a b^{2} a^{2 k}$ by (a).

$r \geqq 1$ : Suppose that $1 \leqq r \leqq k-1$ and the assertion holds for $r$.

$$
\begin{aligned}
(b a)^{k+1} & =(b a)^{k-r+1} a b^{2 r} a^{2 k}=(b a)^{k-r} b a^{2} b^{2 r} a^{2 k} \\
& =(b a)^{k-r} a b^{2} b^{2 r} a^{2 k}=(b a)^{k-(r+1)+1} a b^{2(r+1)} a^{2 k} .
\end{aligned}
$$

This completes the induction. If we then take $k=r$ we obtain

$$
(b a)^{k+1}=b a^{2} b^{2 k} a^{2 k}=a b^{2} b^{2 k} a^{2 k}=a b a^{2 k} .
$$

(d) By (b) and (c) we get

$$
a b=(b a)^{k+1} a=\left(a b a^{2 k}\right) a=a b a^{2 k+1} .
$$


(e) $b a=a b^{2} a^{2 k}=a^{2 k+2} b^{2} a^{2 k}=a^{2 k+1} a b^{2} a^{2 k}=a^{2 k+1} b a$ by (a).

(f) $a b^{2 k+1}=a b b^{2 k}=\left(b^{2 k+1} a b\right) b^{2 k}=b^{2 k+1} a b^{2 k+1}=b^{2 k}\left(b a b^{2 k+1}\right)=b^{2 k} b a=b^{2 k+1} a$.

(g) Note that $\mathrm{C} 1$ and $\mathrm{C} 2$ are satisfied so $e=a^{n} b^{n}$ is the identity of the group kernel $I=S e$. Let $A=\left\{a, a^{2}, \ldots, a^{n}\right\}$ and $B=\left\{b, b^{2}, \ldots, b^{n}\right\}$. Then by Theorem $1.4 S=A \cup B \cup I$ and consequently $|S|=2 n+|G|$. But using the results of [6] we know that $|G|=n g_{n}$ and so we obtain

$$
|S|=2 n+n g_{n}
$$

as required.

All the semigroup presentations we have studied so far have group kernels. However not every semigroup presentation has a group kernel. In fact the next part of Theorem 2.4 gives an example of a presentation $P$ such that $G p P$ is abelian, $S=S m g p P$ is finite but $S$ is not abelian and $S$ has no group kernel.

Example 2.6. Let $P=\left\langle a, b: a^{3}=a, b^{3}=b, a b^{2}=b a^{2}\right\rangle, S=\operatorname{Smgp} P$. Then

(a) $G=G p P$ is isomorphic to $\mathbb{Z}_{2}$.

(b) $S$ has no group kernel, $|S|=10$ and $S$ is not abelian.

Proof. (a) We have

$$
G=G p\left\langle a, b: a^{2}=1, b^{2}=1, a b^{2}=b a^{2}\right\rangle=G p\left\langle a, b: a^{2}=1, b^{2}=1, a=b\right\rangle .
$$

Hence $G p P$ is isomorphic to $\mathbb{Z}_{2}$.

(b) Let $H=\left\{a, a^{2}, b, b^{2}, a b, b a, a b^{2}, b a b, a b a, a^{2} b^{2}\right\}$. We have the following identities:

$$
\begin{gathered}
b^{2} a=b^{2} a^{3}=b b a^{2} a=b a b^{2} a=b a^{2} a b^{2} a=a b^{2} a b^{2} a=a b^{2} b a^{2} a=a b a \\
b a a=a b^{2} \\
a b^{2} a=b a^{2} a=b a \\
b a b a=b a b a^{2} a=b a a b^{2} a=b a^{2} b^{2} a=a b^{2} b^{2} a=a b^{2} a=b a^{2} a=b a \\
a b a a=a b a^{2}=a b^{2} b a^{2}=b a^{2} a b^{2}=b a b^{2} \\
a^{2} b^{2} a=a^{2}(a b a)=a b a \\
a^{2} b^{2}=a b a^{2}=a b^{2} b a^{2}=b a^{2} a b^{2}=b a b^{2}=b^{2} a^{2} .
\end{gathered}
$$

It follows that $H a$ is contained in $H$. The last identity shows that $b^{2} a^{2}$ is an element of $H$. Thus interchanging $a$ with $b$ in $H$ leaves $H$ invariant. Hence $H b$ is also contained in 
$H$. Since $a$ and $b$ are in $H$, then $H=S$, by Theorem 1.6. Now let $X=\{1,2, \ldots, 10\}$. Define the functions $a$ and $b$ from $X$ to $X$ as follows:

$$
\begin{array}{rrrrrrrrrrrrrrrrrrrr}
1 & 2 & 3 & 4 & 5 & 6 & 7 & 8 & 9 & 10 & 1 & 2 & 3 & 4 & 5 & 6 & 7 & 8 & 9 & 10 \\
a: \downarrow & \downarrow & \downarrow & \downarrow & \downarrow & \downarrow & \downarrow & \downarrow & \downarrow & \downarrow & b: \downarrow & \downarrow & \downarrow & \downarrow & \downarrow & \downarrow & \downarrow & \downarrow & \downarrow & \downarrow \\
2 & 1 & 6 & 9 & 9 & 7 & 6 & 6 & 10 & 9 & 5 & 8 & 4 & 3 & 7 & 8 & 5 & 10 & 5 & 8
\end{array}
$$

It is routine to check that $a$ and $b$ satisfy the given relations and all elements in $H$ yield distinct elements. Thus $|S|=10$ and $S$ is non-abelian. It is easy to check that $S$ has no group kernel using this representation of $S$.

Example 2.7. Let $P=\left\langle a, b: a^{5}=a, b^{5}=b, a b^{2}=b a^{2}\right\rangle, S=\operatorname{Smgp} P$. Then

(a) $G=G p P$ has order 20 .

(b) The idempotents of $S$ are $a^{4}, b^{4},(a b)^{2},(b a)^{2}, a^{4} b^{4}$.

(c) The idempotents form a subsemigroup.

(d) $S$ has no group kernel.

(e) $|S|=68$ and $S$ is not abelian.

Proof. The result (a) can be deduced from the results in [1]. We leave the other details to the reader.

Note that the proof of Theorem 2.4 is now complete.

Each of the presentations given above has at least one relation of the form $x^{m}=x$. But as the following theorem shows this not essential for $\operatorname{Smgp} P$ to have finite order.

Theorem 2.8. Let $P=\left\langle a, b: a^{2}=b^{k}, b a b=a\right\rangle, S=\operatorname{Smgp} P$ and $G=G p P$. Then $S a^{4}$ is the group kernel in $S$ and $|S|=k-1+|G|=5 k-1$, where $k \in \mathbb{N}$ and $2 \leqq k$.

Proof. Since $a^{2}=b^{k}$, then $a^{2}$ and $a^{4}$ are central in $S$. It follows that $S a^{4}$ is an ideal in $S$. Also $a^{5}=a^{2} a a^{2}=b^{k} a b^{k}=a$. Thus $e=a^{4}$ is an idempotent in $S$. We also have

$$
(a e)^{4}=a^{4}=e \quad \text { and } \quad(b e)^{2 k}=b^{2 k} a^{8 k}=a^{4} a^{4}=a^{4}=e .
$$

Hence $a e$ and $b e$ have inverses in $S e$. This shows that $I=S e$ is the group kernel in $S$. Let $B=\left\{b, \ldots, b^{k-1}\right\}$. Then by Theorem $1.5, \operatorname{In} B=\varnothing$ and $b, \ldots, b^{k-1}$ are pairwise distinct. If $w$ is a word in $S \backslash B$, then $w$ is of the form $w=\ldots a \ldots$ and so $w=\ldots a a^{4} \ldots=w e$ belongs to $I$. Consequently $S=B \cup I$. If we let

$$
x=b, y=a, n=2, s=k, m=2 k, r=2 k-1,
$$

we see that $G$ is isomorphic to a metacyclic group and so $|G|=2 \cdot 2 k=4 k$. Hence $|S|=5 k-1$.

\section{Description of the computer program}

The computer program is an implementation based loosely on the method described 
by Neumann in [5] and incorporates some ideas from [2]. To describe the program we choose a concrete example and explain the main ideas using this example.

The program has a few main variables which we will list below in an effort to make the description of the program clearer.

Def List : A doubly linked list used to keep track of definitions made.

DefTable: A two-dimensional array of integers. DefTable $[i, j]$ is the definition made for $i * j$ where $j$ is one of the generators. If no definition is made for $i * j$, then Def Table $[i, j]=0$.

EquClass: A one-dimensional array of integers. The equivalence class $E[i]$ of $i$ can be determined by using the following procedure:

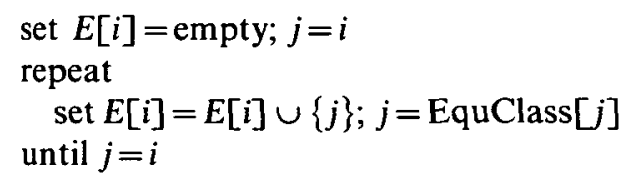

EquPair : A stack, which is an array of equivalence pairs, used to keep track of equivalences.

Let $P=\left\langle X: L_{1}=R_{1}, L_{2}=R_{2}, \ldots, L_{s}=R_{s}\right\rangle$ be a presentation on $X$. We first define 0 to represent the empty word, which of course is not part of the semigroup. Starting with the definition 0 and stepping forward through the list Def List for a given relation $L=R$, where $L=a_{1} a_{2} \ldots a_{n}$ and $R=b_{1} b_{2} \ldots b_{m}$, and a definition $k$, then we process this relation in the following fashion:

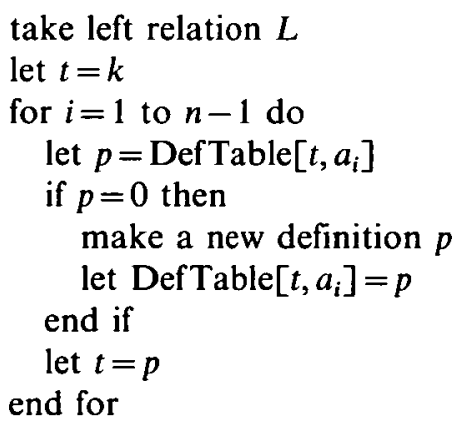

At the end of this process we obtain a definition $l d=t$ waiting to be processed to see whether a definition $l d * a_{n}$ needs to be made.

Repeating the same process for $R$ we obtain $r d$ waiting to be processed to see whether a definition $r d * b_{m}$ needs to be made. There are four cases that may occur.

Case 1. One of the integers DefTable $\left[l d, a_{n}\right]$ and DefTable $\left[r d, b_{m}\right]$ is zero and the other is not. In this case we set the zero one to be the same as the non-zero one.

Case 2. DefTable $\left[l d, a_{n}\right]$ and DefTable $\left[r d, b_{m}\right]$ are both non-zero and equal. In this case we continue the above process with the next relation. 
Case 3. DefTable $\left[l d, a_{n}\right]$ and DefTable $\left[r d, b_{m}\right]$ are both zero. In this case we reprocess the left relation. This is necessary if $L$ is embedded in $R$. After this we may end up with case 1 or case 3 again. If we end up with case 3 , we make a new definition $p$, and set both of DefTable $\left[l d, a_{n}\right]$ and DefTable $\left[r d, b_{m}\right]$ equal to $p$.

Case 4. $c=\operatorname{DefTable}\left[l d, a_{n}\right]$ and $d=\operatorname{DefTable}\left[r d, b_{m}\right]$ are both non-zero but $c \neq d$. This means that $k * L=c$ and $k * R=d$ yield distinct results. Since this is impossible $c$ and $d$ must be equivalent. To resolve this problem we proceed as follows.

for each definition $k$ let EquClass $[k]=k$

push the pair $(c, d)$ into the stack EquPair

while stack EquPair is not empty do

pop pair $(a, b)$ from the stack

for each generator $g$ do

let $\mu=\operatorname{DefTable}[a, g]$ and $v=\operatorname{DefTable}[b, g]$

if $\mu \neq 0$ and $\nu=0$ then let DefTable $[b, g]=\mu$

if $\mu=0$ and $\nu \neq 0$ then let DefTable $[a, g]=v$

if both $\mu \neq 0, v \neq 0$ and $\mu \neq v$ then end for

if $\mu$ is not equivalent to $v$, then push the pair $(\mu, v)$ into the stack

join the equivalence classes of $a$ and $b$

end while

for each equivalence class $E[i]$ with more than two elements, replace each occurrence of $x \in E[i] \backslash\{i\}$ with $i$ in the Def Table.

Remove $x$ from DefList.

Now restart the process with the next definition which is not yet processed until all the definitions in the DefList are finished. If the program terminates in a finite number of steps then the total number of definitions is one more than the order of the semigroup.

Consider the semigroup $S$ given in Example 2.6. Using this example, we list below the tables which show how definitions are made and equivalences resolved by the computer program described above.

We define 0 to be the empty word, 1 to be $a$, and 2 to be $b$. We process each relation making definitions as described above. An asterisk denotes an equivalence.

\begin{tabular}{lllllllllllllllllllll}
\multicolumn{1}{c}{$a$} & $a$ & $a$ & $a$ & & \multicolumn{1}{c}{$b$} & \multicolumn{1}{c}{$b$} & $b$ & \multicolumn{1}{c}{$b$} & \multicolumn{3}{c}{$a$} & $b$ & $b$ & $=$ & $b$ & $a$ & $a$ \\
0 & 1 & 3 & 1 & 0 & 1 & 0 & 2 & 4 & 2 & 0 & 2 & 0 & 1 & 5 & 7 & 0 & 2 & 6 & 7 \\
1 & 3 & 1 & 3 & 1 & 3 & 1 & 5 & 7 & 5 & 1 & 5 & 1 & 3 & 8 & 10 & 1 & 5 & 9 & 10 \\
2 & 6 & 7 & 6 & 2 & 6 & 2 & 4 & 2 & 4 & 2 & 4 & 2 & 6 & 11 & 13 & 2 & 4 & 12 & 13 \\
3 & 1 & 3 & 1 & 3 & 1 & 3 & 8 & 10 & 8 & 3 & 8 & 3 & 1 & 5 & 7 & 3 & 8 & 14 & 7 \\
4 & 12 & 13 & 12 & 4 & 12 & 4 & 2 & 4 & 2 & 4 & 2 & 4 & 12 & 15 & 7 & 4 & 2 & 6 & 7 \\
5 & 9 & 10 & 9 & 5 & 9 & 5 & 7 & 5 & 7 & 5 & 7 & 5 & 9 & 16 & 7 & 5 & 7 & 6 & 7 \\
6 & 7 & 6 & 7 & 6 & 7 & 6 & 11 & 13 & 11 & 6 & 11 & 6 & 7 & 5 & 7 & 6 & 11 & 17 & 7 \\
7 & 6 & 7 & 6 & 7 & 6 & 7 & 5 & 7 & 5 & 7 & 5 & 7 & 6 & 11 & $13^{*}$ & 7 & 5 & 9 & $10^{*}$
\end{tabular}


After processing relation 3 , we find that 13 and 10 are equivalent. Before processing this equivalence relation the linked list of definitions is as follows:

$\begin{array}{llllllllllllllllll}\text { DefList: } 0 & 1 & 2 & 3 & 4 & 5 & 6 & 7 & 8 & 9 & 10 & 11 & 12 & 13 & 14 & 15 & 16 & 17 .\end{array}$

This equivalence yields the following non-trivial equivalence classes:

Class of $8=\{8,11\}$

Class of $9=\{9,12\}$

Class of $10=\{10,13\}$

Class of $14=\{14,17\}$

Class of $15=\{15,16\}$

After processing these equivalences, the linked list of definitions reduces to

$$
\text { Def List: } 0 \begin{array}{lllllllllllll}
0 & 1 & 2 & 3 & 4 & 5 & 6 & 7 & 8 & 9 & 10 & 14 & 15 .
\end{array}
$$

If we compare the old and new DefList, we see that they are the same up to definition 6 and the program paused to resolve the equivalence $13=10$ while processing definition 7. So we only need to make a partial table to describe how the program continues starting with definition 7 . But we list below the whole table starting with definition 0 , to show how all necessary changes are made and how all the equivalences are resolved. Later we will list only the partial tables which start with the definition which is not yet completely processed.

\begin{tabular}{llllllllllllllllllllll}
\multicolumn{1}{c}{$a$} & $a$ & $a$ & $=$ & $a$ & \multicolumn{1}{c}{$b$} & $b$ & $b$ & $=$ & $b$ & & $a$ & $b$ & $b$ & $=$ & $b$ & $a$ & $a$ \\
0 & 1 & 3 & 1 & 0 & 1 & 0 & 2 & 4 & 2 & 0 & 2 & 0 & 1 & 5 & 7 & 0 & 2 & 6 & 7 \\
1 & 3 & 1 & 3 & 1 & 3 & 1 & 5 & 7 & 5 & 1 & 5 & 1 & 3 & 8 & 10 & 1 & 5 & 9 & 10 \\
2 & 6 & 7 & 6 & 2 & 6 & 2 & 4 & 2 & 4 & 2 & 4 & 2 & 6 & 8 & 10 & 2 & 4 & 9 & 10 \\
3 & 1 & 3 & 1 & 3 & 1 & 3 & 8 & 10 & 8 & 3 & 8 & 3 & 1 & 5 & 7 & 3 & 8 & 14 & 7 \\
4 & 9 & 10 & 9 & 4 & 9 & 4 & 2 & 4 & 2 & 4 & 2 & 4 & 9 & 15 & 7 & 4 & 2 & 6 & 7 \\
5 & 9 & 10 & 9 & 5 & 9 & 5 & 7 & 5 & 7 & 5 & 7 & 5 & 9 & 15 & 7 & 5 & 7 & 6 & 7 \\
6 & 7 & 6 & 7 & 6 & 7 & 6 & 8 & 10 & 8 & 6 & 8 & 6 & 7 & 5 & 7 & 6 & 8 & 14 & 7 \\
7 & 6 & 7 & 6 & 7 & 6 & 7 & 5 & 7 & 5 & 7 & 5 & 7 & 6 & 8 & 10 & 7 & 5 & 9 & 10 \\
8 & 14 & 7 & $6 *$ & 8 & $14 *$ & & & & & & & & & & & & &
\end{tabular}

After processing relation 1 , we find that 6 and 14 are equivalent. Before processing this equivalence relation the linked list Def List of definitions is as follows:

$$
\begin{array}{lllllllllllll}
\text { Def List: } 0 & 1 & 2 & 3 & 4 & 5 & 6 & 7 & 8 & 9 & 10 & 14 & 15 .
\end{array}
$$

This equivalence yields only one non-trivial equivalence class $\{6,14\}$. After processing this equivalence, the linked list of definitions reduces to 
Def List: $\begin{array}{llllllllllll}0 & 1 & 2 & 3 & 4 & 5 & 6 & 7 & 8 & 9 & 10 & 15 .\end{array}$

We continue with definition 8 and obtain

$$
\begin{array}{ccccccccccccccccccccc}
\multicolumn{4}{c}{a} & a & a & = & a & \multicolumn{1}{c}{b} & b & b & = & b & & a & b & b & = & b & a & a \\
8 & 6 & 7 & 6 & 8 & 6 & 8 & 10 & 8 & 10 & 8 & 10 & 8 & 6 & 8 & 10 & 8 & 10 & 9 & 10 \\
9 & 10 & 9 & 10 & 9 & 10 & 9 & 15 & 7 & 5^{*} & 9 & 15^{*} & & & & & & &
\end{array}
$$

While processing relation 2, we find that 5 and 15 are equivalent. Before processing this equivalence relation the linked list of definitions is as follows:

$$
\text { Def List: } \begin{array}{llllllllllll}
0 & 1 & 2 & 3 & 4 & 5 & 6 & 7 & 8 & 9 & 10 & 15 .
\end{array}
$$

This equivalence yields only one non-trivial equivalence class $\{5,15\}$. After processing this equivalence, the linked list of definitions reduces to:

$$
\text { DefList: } \begin{array}{lllllllllll}
0 & 1 & 2 & 3 & 4 & 5 & 6 & 7 & 8 & 9 & 10 .
\end{array}
$$

We continue with definition 9 and finally end up with the following consistent table:

\begin{tabular}{llllllllllllllllllllll}
\multicolumn{1}{c}{$a$} & $a$ & \multicolumn{1}{c}{$a$} & \multicolumn{1}{c}{$a$} & \multicolumn{1}{c}{$b$} & \multicolumn{1}{c}{$b$} & \multicolumn{1}{c}{$b$} & \multicolumn{4}{c}{$a$} & $b$ & $b$ & $b$ & $a$ & $a$ \\
0 & 1 & 3 & 1 & 0 & 1 & 0 & 2 & 4 & 2 & 0 & 2 & 0 & 1 & 5 & 7 & 0 & 2 & 6 & 7 \\
1 & 3 & 1 & 3 & 1 & 3 & 1 & 5 & 7 & 5 & 1 & 5 & 1 & 3 & 8 & 10 & 1 & 5 & 9 & 10 \\
2 & 6 & 7 & 6 & 2 & 6 & 2 & 4 & 2 & 4 & 2 & 4 & 2 & 6 & 8 & 10 & 2 & 4 & 9 & 10 \\
3 & 1 & 3 & 1 & 3 & 1 & 3 & 8 & 10 & 8 & 3 & 8 & 3 & 1 & 5 & 7 & 3 & 8 & 6 & 7 \\
4 & 9 & 10 & 9 & 4 & 9 & 4 & 2 & 4 & 2 & 4 & 2 & 4 & 9 & 5 & 7 & 4 & 2 & 6 & 7 \\
5 & 9 & 10 & 9 & 5 & 9 & 5 & 7 & 5 & 7 & 5 & 7 & 5 & 9 & 5 & 7 & 5 & 7 & 6 & 7 \\
6 & 7 & 6 & 7 & 6 & 7 & 6 & 8 & 10 & 8 & 6 & 8 & 6 & 7 & 5 & 7 & 6 & 8 & 6 & 7 \\
7 & 6 & 7 & 6 & 7 & 6 & 7 & 5 & 7 & 5 & 7 & 5 & 7 & 6 & 8 & 10 & 7 & 5 & 9 & 10 \\
8 & 6 & 7 & 6 & 8 & 6 & 8 & 10 & 8 & 10 & 8 & 10 & 8 & 6 & 8 & 10 & 8 & 10 & 9 & 10 \\
9 & 10 & 9 & 10 & 9 & 10 & 9 & 5 & 7 & 5 & 9 & 5 & 9 & 10 & 8 & 10 & 9 & 5 & 9 & 10 \\
10 & 9 & 10 & 9 & 10 & 9 & 10 & 8 & 10 & 8 & 10 & 8 & 10 & 9 & 5 & 7 & 10 & 8 & 6 & 7
\end{tabular}

As is proved in Example 2.6, the semigroup $S$ has order 10 .

Acknowledgements. The authors would like to thank C. M. Campbell and J. Jones for helpful conversations. The second author would like to thank the Royal Society and the British Council for financial support while this work was being carried out.

\section{REFERENCES}

1. C. M. Campbell and R. M. Thomas, On $(2, n)$-groups related to Fibonacci groups, Israel $J$. Math. 58 (1987), 370-380. 
2. J. J. Cannon, L. A. Dimino, G. Havas and J. M. Watson, Implementation and analysis of the Todd-Coxeter algorithm, Math. Comp. 27 (1973), 463-490.

3. A. Jura, Coset enumeration in a finitely presented semigroup, Canad. Math. Bull. 21 (1978), $37-46$.

4. J. Leech, Coset enumeration on digital computers, Proc. Cambridge Philos. Soc. 59 (1963), 257-267.

5. B. H. Neumann, Some remarks on semigroup presentations, Canad. J. Math. 19 (1967), 1018-1026.

6. E. F. Robertson and C. M. CAmpbell, Symmetric presentations, in Group theory, Proceedings of the 1987 Singapore Conference (Walter de Gruyter, Berlin, New York 1989), 497-506.

7. J. A. Todd and H. S. M. Coxeter, A practical method for enumerating cosets of a finite abstract group, Proc. Edinburgh Math. Soc. 5 (1936), 26-34.

UNIVERSITY OF ST ANDREWS

St ANDREwS KY16 9SS

SCOTLAND
Çukurova Üniversitesi BALCALI

Adana

TURKEY 